\title{
Fast and Accurate Connectivity Analysis Between Functional Regions Based on DT-MRI
}

\author{
Dorit Merhof $^{1,2, \star}$, Mirco Richter ${ }^{1}$, Frank Enders ${ }^{1,2}$, Peter Hastreiter ${ }^{1,2}$, \\ Oliver Ganslandt ${ }^{2}$, Michael Buchfelder ${ }^{2}$, Christopher Nimsky ${ }^{2}$, and Günther Greiner ${ }^{1}$ \\ ${ }^{1}$ Computer Graphics Group, University of Erlangen-Nuremberg, Germany \\ ${ }^{2}$ Neurocenter, Dept. of Neurosurgery, University of Erlangen-Nuremberg, Germany \\ merhof@cs.fau.de
}

\begin{abstract}
Diffusion tensor and functional MRI data provide insight into function and structure of the human brain. However, connectivity analysis between functional areas is still a challenge when using traditional fiber tracking techniques. For this reason, alternative approaches incorporating the entire tensor information have emerged. Based on previous research employing pathfinding for connectivity analysis, we present a novel search grid and an improved cost function which essentially contributes to more precise paths. Additionally, implementation aspects are considered making connectivity analysis very efficient which is crucial for surgery planning. In comparison to other algorithms, the presented technique is by far faster while providing connections of comparable quality. The clinical relevance is demonstrated by reconstructed connections between motor and sensory speech areas in patients with lesions located in between.
\end{abstract}

\section{Introduction}

In recent years, medical imaging techniques such as diffusion tensor imaging (DTI) and functional MRI (fMRI) have emerged enabling the exploration of function and structure of the human brain. DTI measures the diffusion of water which is anisotropic in tissue with a high degree of directional organization. For the computation of diffusion tensors, diffusion-weighted images for at least six non-collinear gradient directions are acquired. The respective diffusion tensors provide information about the location and orientation of white matter structures in vivo. The localization of active brain areas such as motor and sensory speech areas is accomplished with fMRI.

In neurosurgery, the localization of functional areas on the cortex and their white matter connectivity is of great importance for preoperative planning. With respect to the motor and sensory speech areas, the Broca's and Wernicke's areas located in the cerebral cortex, it is generally accepted that these areas are functionally related in speech processing. To avoid neurological deficits after neurosurgical procedures, eloquent structures such as the speech areas as well as connecting white matter structures have to remain intact. For this reason, analysis of white matter connectivity between functional areas is of high interest for neurosurgery and other disciplines in neuroscience.

Standard techniques for the reconstruction of white matter structures from DTI data rely on the orientation of the major eigenvector of the diffusion tensor [1]. Thereby,

\footnotetext{
${ }^{\star}$ Corresponding author.
} 
streamline-based techniques are employed to propagate the fiber. These approaches enable the reconstruction of major tract systems such as the pyramidal tract or the corpus callosum. However, the reliability of standard tracking techniques is affected by imaging noise and partial volume effects in case of crossing or branching fibers.

For this reason, standard tracking algorithms are not suited for a connectivity analysis involving sub-cortical or cortical regions. First approaches addressing this problem used probabilistic or regularization techniques [2]3]. Over a large number of iterations, the process yields a connectivity probability related to the number of probabilistic streamlines found in a volume element. Another class of algorithms derived from level set theory considers arrival times of diffusion fronts [45]. In most recent work based on level sets, the problem of white matter connectivity is modeled as wavefront evolution based on a cost function which depends on the entire diffusion tensor [5]. Considering the arrival times of the wavefront, connections are derived by minimizing the cumulative travel cost along the path. Another recent approach uses global optimization and dynamic programming for fiber reconstruction [6]. A graph is spanned over the domain with assigned cost for each edge connecting two voxels. By means of dynamic programming, connections with highest probability are computed.

In this work, we extend our previous research for connectivity analysis based on pathfinding [7]. Basically, pathfinding algorithms are highly efficient techniques commonly used in artificial intelligence for problems associated with a state space search using cost functions. They are applied to derive the minimum-cost path between a start and a target region. To investigate neuronal connectivity, a cost function based on the probability distribution function of each tensor is employed. Similarly to other connectivity algorithms [56], the entire tensor controls the pathfinding procedure to circumvent biasing of the major eigenvector in isotropic regions. We present several crucial enhancements of our basic algorithm such as an improved cost function, a novel search grid and an optimized implementation. As an important result for clinical application, the presented algorithm is considerably faster than other recently presented connectivity algorithms and provides at the same time comparable accuracy.

\section{Method}

Pathfinding algorithms are commonly used in computer science for different types of search problems. Since there are highly efficient solutions such as the A* algorithm [8], it is straightforward to apply pathfinding in the context of white matter connectivity analysis which can be considered as an instance of a minimum-cost path problem [567].

\subsection{Pathfinding}

The $\mathrm{A}^{*}$ algorithm was designed to efficiently compute the path with lowest cost between a start and a target region. For this purpose, the algorithm builds up a graph with nodes and edges, where the edges are assigned a local cost. In each iteration, the path with lowest cost is expanded until the target region is reached. An important fact about the $\mathrm{A}^{*}$ algorithm is its optimality [9] which guarantees that the best possible solution is found with the smallest computational effort. 
For performing the search, the algorithm maintains two lists, an open list comprising all nodes currently under consideration and a closed list containing nodes that have already been processed. In the beginning, the open list comprises all nodes from the start region and the closed list is empty. Each node stores the movement cost $g_{i}$ required to travel along the path to the respective node $i$. The cost function $f_{i}=g_{i}+h_{i}$ is evaluated for each node of the open list to decide which one to process next. Thereby, $h_{i}$ denotes an estimate of the remaining cost (also called heuristic) to the target which may optionally be added to direct the search towards the target making the algorithm more efficient. Otherwise, the search would equally spread in all directions. It is important to note that $h_{i}$ does not affect the optimality of $\mathrm{A}^{*}$ as long as $h_{i}$ is not higher than the actual cost necessary to reach the target.

To compute the minimum cost path, the algorithm repeatedly selects the node with lowest $f_{i}$ from the open list, adds all its neighbors to the open list and moves the selected node to the closed list. These processing steps continue until the target node is added to the open list and the path with the lowest cost is found.

\subsection{Grid for Partitioning the Search Space}

For navigating in three-dimensional space, the search algorithm requires a grid which should provide a regular structure uniformly covering the search space and a small step-size between neighbor nodes. The angle between edges should be small to provide enough flexibility to follow the direction of anisotropic diffusion. For this reason, we use a hexahedral grid connecting each node to 74 neighbors as shown in Figure 1 (left). In comparison to a grid with 26 neighbors, further directions are provided reducing the angle between neighbor edges and offering a considerably improved choice of different directions thus fulfilling the flexibility criterion. The grid size has to be chosen sufficiently small to guarantee a small maximum step-size which should not exceed $2 \mathrm{~mm}$ which is the grid size of the DTI dataset. The resulting grid is of high resolution and enables a dense sampling of the search space as outlined in Figure 11(middle, right).
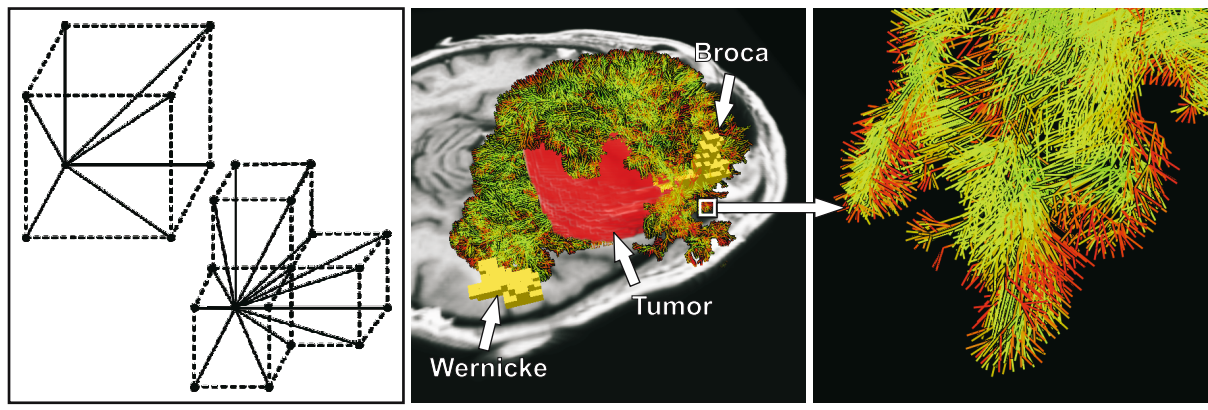

Fig. 1. Octant of a grid with 26 and 74 neighbors (left). Expansion of the search space between Broca's and Wernicke's speech areas using a grid with 74 neighbors and a maximum edge length of $1.5 \mathrm{~mm}$ in a brain tumor patient (middle). Close-up view showing details of search grid (right). 


\subsection{Cost Function}

The cost function is an integral part for all types of connectivity algorithms since it controls the process of path evolution. To overcome the limitations encountered with fiber tracking arising from the reduction of the tensor information to the principal eigenvector, the cost function for connectivity algorithms has to incorporate the entire tensor information. For this reason, the surface of the tensor ellipsoid, with half-axes aligned to the eigenvectors of the tensor and scaled according to the length of the respective eigenvalues $\lambda_{i, i=1,2,3}$, is commonly used as probability profile [56]7]. Thereby, the probability of a fiber following a certain direction corresponds to the distance between the center of the ellipsoid located at $(0,0,0)^{T}$ and the intersection point $\boldsymbol{r}$ on the surface of the ellipsoid. To obtain probabilities between 0 and 1, the tensor ellipsoid is normalized using $\lambda_{1}$ resulting in a maximum length of 1 for any segment connecting the center of the ellipsoid with its surface. As a result, the diffusion probability $p_{i}(\boldsymbol{r})$ for any direction can be directly obtained from the profile of the normalized ellipsoid:

$$
p_{i}(\boldsymbol{r})=\frac{\|\boldsymbol{r}\|}{\lambda_{1}} .
$$

However, the resulting probability profile results in a bias towards spherical ellipsoids since they are traversed more easily due to a high probability for all directions. In [5], this is addressed by incorporating fractional anisotropy (FA) [10] into the cost function. In this work, we propose to model the anisotropic characteristic of a tensor by subtracting the isotropic part represented by $\lambda_{3}$ before normalization:

$$
p_{i}(\boldsymbol{r})=\frac{\|\boldsymbol{r}\|-\lambda_{3}}{\lambda_{1}} .
$$

This is motivated by considering the probability profile of the tensor. In Figure 2 the probability profiles for a linear and a spherical tensor are plotted as a function of the azimuthal and polar angle of the corresponding ellipsoid. In case of Equation 1 (left plot in each section), the almost spherical tensor yields very high probabilities for all directions resulting in a bias of isotropic tensors. This is circumvented by the probability profile resulting from Equation 2 (right plot in each section) which on the one hand perfectly maintains the shape of the probability profile but cuts the isotropic fraction. In this way, the probability function is solely based on the tensor probability profile and no additional term in the cost function is necessary. The resulting cost function is thus defined as $c_{i}(\boldsymbol{r})=1-p_{i}(\boldsymbol{r})$ with $p_{i}(\boldsymbol{r})$ derived from Equation 2 Based on this approach, a more comprehensive probability profile is provided which better captures the tensor properties making the incorporation of FA into the cost function redundant.

\subsection{Minimum Cost Path}

Based on the cost function, minimum-cost connections representing neuronal structures are derived. For this purpose, minimum-cost paths are determined by summing up all local costs $c_{j}$ encountered along the path to node $i$ resulting in a global cost $g_{i}$ for the whole path [5]67]: 


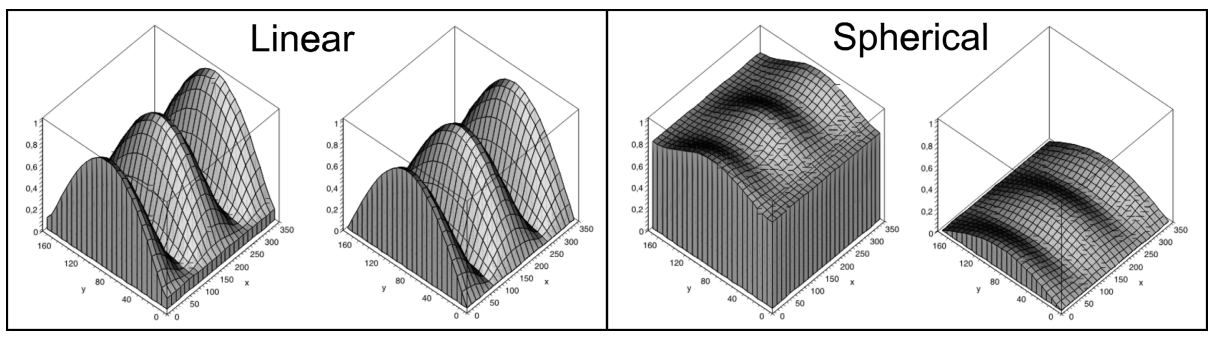

Fig. 2. Tensor probability profiles (z-axis) of a linear and an almost spherical tensor for Eq.1 (left in each section) and Eq. 2 (right in each section), plotted as a function of the azimuthal and polar angle ( $\mathrm{x}$ - and $\mathrm{y}$-axis) of the corresponding ellipsoid.

$$
g_{i}=\sum_{j=1}^{i} c_{j} \rightarrow \min .
$$

In this way, connections are derived fulfilling the global optimum condition. Thereby, the pathfinding algorithm preferably propagates paths with low global cost by comparing the cost of all nodes on the open list. As a result, the computed connections between all voxels of the start region and the target region are guaranteed to be optimal.

\subsection{Efficient Search}

To ensure that the search is oriented towards the target, the $\mathrm{A}^{*}$ algorithm takes advantage of an estimate $h_{i}$ of the remaining cost (heuristic). If no heuristic is used, the algorithm expands equally in all directions resulting in a greater search space and an increased computational cost. If $h_{i}$ is admissible, i.e. it never overestimates the cost to the target, then $\mathrm{A}^{*}$ is guaranteed to find the path with lowest cost. For this purpose, a gradient $h_{i}$ is employed in the cost function $f_{i}=g_{i}+h_{i}$ to direct the search towards the target:

$$
h_{i}=\frac{d_{i}}{s_{\max }} \cdot \hat{\bar{c}}
$$

Thereby, $d_{i}$ denotes the Euclidean distance of node $i$ to the target which is normalized according to the maximum step length $s_{\max }$ within the grid. The minimum number of steps to the target $d_{i} / s_{\max }$ is multiplied with the estimated average cost per step $\hat{\bar{c}}$ which is determined by sampling the data for short connections in regions with high FA. The smallest cost among the samples is then assigned to $\hat{\bar{c}}$. This additional term of the cost function $f_{i}$ directs the search towards the target. According to our observations, it can be empirically approved that the heuristic is admissible since the resulting paths did not differ from paths computed without heuristic. The computing time for the search could be reduced considerably which is outlined in more detail in Section 3 .

\section{Results and Discussion}

For evaluation purposes, two proband and three patient DTI datasets (voxel size: $1.875 \times 1.875 \times 1.9 \mathrm{~mm}^{3}, 128 \times 128 \times 60$ voxels $)$ were acquired with a Siemens Sonata 
1.5 Tesla scanner. For all datasets, seed regions corresponding to the Broca's and Wernicke's speech areas derived from fMRI were available. All computations were performed on a PC equipped with an Intel Pentium 4, $3.4 \mathrm{GHz}$, and 2 GB RAM. For pathfinding, we used the 74-neighbor grid (see Section 2.2) with a maximum step length of $1.5 \mathrm{~mm}$. Similarly to [567], we emploed a FA threshold $T_{F A}$ to restrict the search to regions of white matter only. In all our experiments, $T_{F A}$ amounted to 0.3 excluding nodes with an FA value below the threshold from further processing. Our evaluation investigates the quality of the obtained paths, the computing time is analyzed and a comparison with other techniques is drawn.

Quality Analysis. To investigate the accuracy of the proposed cost function (Equation 2) in comparison to a cost function based on the product of the normalized probability profile (Equation 1) and the local FA value [5], we employed the validity index introduced by Jackowski [5]. The validity index computes the scalar product between path tangent and major eigenvector for each segment and returns the average value for the whole path. Accordingly, we also recorded the average probability according to the probability profile of Equation 2 and the average FA value for each path. Table 1 shows the minimum, maximum and average value for the connections derived between speech areas in two of our datasets. As a result, our cost function achieves better results compared to the normalized tensor profile commonly employed [567]. In addition to that, our approach reaches equal accuracy with respect to the validity index compared to wavefront evolution [5].

Table 1. Evaluation based on validity index, probability profile and FA represented by average, maximum and minimum value of all fibers

\begin{tabular}{|c|c|c|c|c|c|c|c|c|c|c|c|c|}
\hline Cost Function & \multicolumn{6}{|c|}{ Equation 1, FA } & \multicolumn{6}{|c|}{ Equation 2} \\
\hline & \multicolumn{3}{|c|}{ Patient 1} & \multicolumn{3}{|c|}{ Patient 2} & \multicolumn{3}{|c|}{ Patient 1} & \multicolumn{3}{|c|}{ Patient 2} \\
\hline Number of fibers & \multicolumn{3}{|c|}{17} & \multicolumn{3}{|c|}{21} & \multicolumn{3}{|c|}{19} & \multicolumn{3}{|c|}{23} \\
\hline \multirow[b]{2}{*}{ Validity Index } & $a v g$ & $\min$ & $\max$ & $a v g$ & $\min$ & $\max$ & $a v g$ & $\min$ & $\max$ & avg & $\min$ & $\max$ \\
\hline & 0.75 & 0.69 & 0.87 & 0.83 & 0.82 & 0.86 & 0.77 & 0.70 & 0.88 & 0.86 & 0.85 & 0.87 \\
\hline Probability Profile & 0.68 & 0.65 & 0.70 & 0.63 & 0.62 & 0.64 & 0.75 & 0.73 & 0.82 & 0.70 & 0.69 & 0.70 \\
\hline Fractional Anisotropy & 0.58 & 0.54 & 0.61 & 0.56 & 0.55 & 0.57 & 0.61 & 0.57 & 0.68 & 0.56 & 0.55 & 0.57 \\
\hline
\end{tabular}

For illustration, the local probability according to Equation 2 is visualized in each step by color encoding assigning red to a low and green to a high value of the probability profile from Equation 2. In Figure 3, color coding is used to compare standard fiber tracking based on streamline propagation [1] (RK-4 integration, step size $0.5 \mathrm{~mm}$ ) and pathfinding with regard to their exactness. The red segments encountered in case of fiber tracking indicate that spherical tensors were crossed. Contrarily, a more reliable path was obtained using pathfinding which takes into account the entire probability profile of the local tensor which resulted in paths including anisotropic tensors.

Computing Time. Since the algorithm aims at clinical application requiring fast interaction times, computational cost is of major concern in addition to accuracy (see Table 2). Implementation features essentially contributing to high processing times are an 

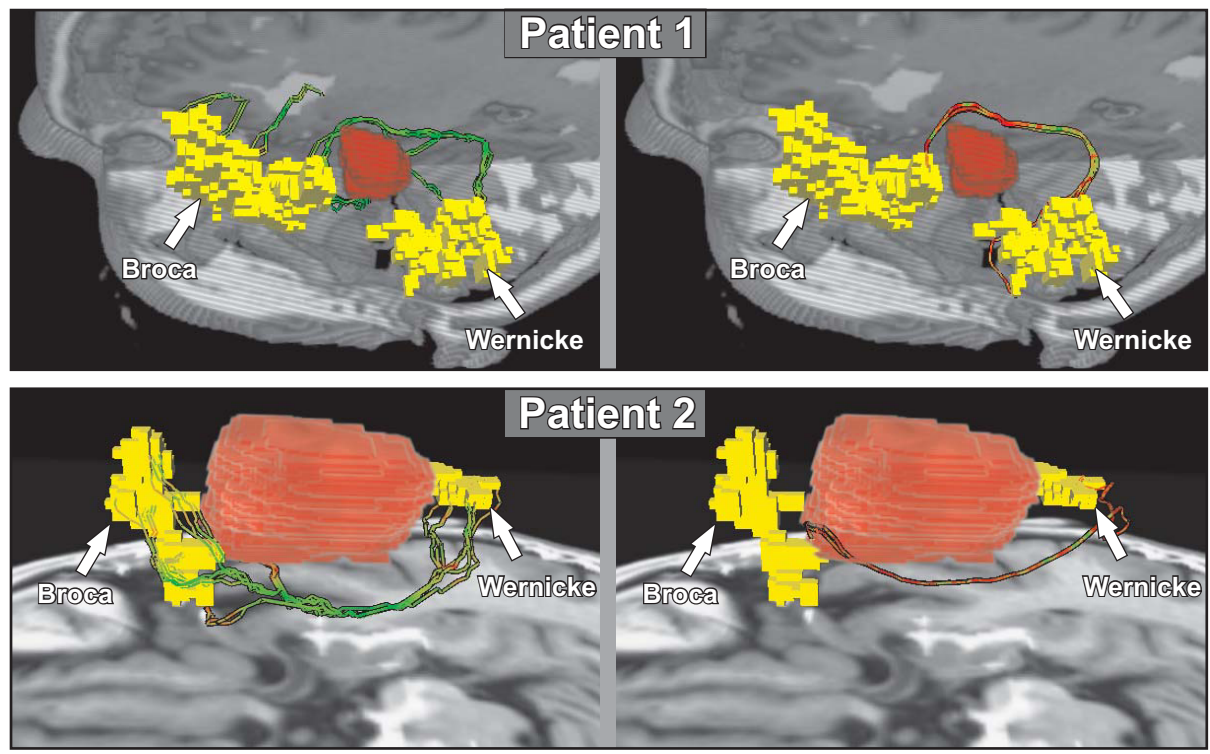

Fig. 3. Upper row (Patient 1): Patient with a cavernoma. Lower row (Patient 2): Patient with a glioblastoma multiforme (WHO grade IV) having speech dominance on the right hemisphere. The respective lesion is shown in red in each patient. Pathfinding (left) vs. fiber tracking (right), color coding shows that pathfinding provides more precise results.

Table 2. Average computing time per path and expansion of search grid (number of nodes) for search based on global cost $g_{i}$ (Eq.2) and speed up encountered with heuristic $h_{i}$

\begin{tabular}{|l|r|r|r|r|}
\hline Performance & \multicolumn{2}{|c|}{ Computing Time } & \multicolumn{2}{c|}{ Number of Grid Nodes } \\
\hline & Patient 1 & Patient 2 & Patient 1 & Patient 2 \\
\hline$f_{i}=g_{i}$ & $42.0 \mathrm{sec}$ & $82.8 \mathrm{sec}$ & 240705 & 318163 \\
$f_{i}=g_{i}+h_{i}$ & $14.9 \mathrm{sec}$ & $19.5 \mathrm{sec}$ & 106414 & 163586 \\
\hline
\end{tabular}

efficient implementation of the open and closed list since they encounter frequent access and have to administrate a high number of nodes. For this reason, we used a combination of buckets and sorted vectors for the open list and a hash map for the closed list. Incorporation of the heuristic resulted in a speed up of approximately $70 \%$, compared to pathfinding solely using the cost function. In each case, the resulting paths remained the same, since the heuristic is admissible according to empirical observations.

Comparison with other Approaches. As outlined in Figure 3, standard fiber tracking is inappropriate for connectivity analysis in subcortical areas requiring alternative approaches. Since it is anticipated that neuronal connections are kept optimal [11], minimum-cost approaches have been developed to model connectivity [5|6|7]. In comparison to the normalized tensor profiles employed in recent work [5]6]7, connectivity results were significantly improved using our novel cost function characterizing both 
shape and anisotropy of the local tensor. From the algorithmic point of view, pathfinding is computationally more efficient than other graph-based techniques such as [6], since it can be proven that no other search algorithm which is guaranteed to find the minimumcost path requires less computational expense than $A^{*}$ [9]. Apart from that, the presented grid structure provides high resolution and a considerably increased number of neighbor nodes to sample the search space very densely which is superior compared to the grid used in [6]. Overall, the presented approach provides comparable or even better accuracy compared to other approaches and is, at the same time, by far faster than other current approaches.

\section{Conclusion and Future Work}

Based on previous work introducing pathfinding for the problem of neuronal connectivity within the human brain, we presented an improved cost function, a high resolution grid for sampling the search space and an efficient implementation enabling interactive application. Accurate paths according to different quality measures were obtained. The approach has several advantages over existing methods, such as highly efficient processing times which is important for clinical application.

Since the quality of the obtained connections would further benefit from tensor field regularization or higher-order tensor representations derived from high angular resolution diffusion images, future work will aim at incorporation of these techniques.

\section{Acknowledgments}

This work was supported by the Deutsche Forschungsgemeinschaft in the context of SFB 603, Project C9. We are especially grateful to Peter Grummich (Dept. of Neurosurgery, University of Erlangen-Nuremberg, Germany) for speech fMRI.

\section{References}

1. Mori, S., Crain, B., Chacko, V., van Zijl, P.: Three-dimensional tracking of axonal projections in the brain by magnetic resonance imaging. Ann Neurol 45 (1999) 265-269

2. Behrens, T., Johansen-Berg, H., Woolrich, M., Smith, S., Wheeler-Kingshott, C., Boulby, P., Barker, G., Sillery, E., Sheehan, K., Ciccarelli, O., Thompson, A., Brady, J., Matthews, P.: Non-invasive mapping of connections between human thalamus and cortex using diffusion imaging. Nat Neurosci 6 (2003) 750-757

3. Björnemo, M., Brun, A., Kikinis, R., Westin, C.F.: Regularized stochastic white matter tractography using diffusion tensor MRI. In: MICCAI. (2002) 435-442

4. O'Donnell, L., Haker, S., Westin, C.F.: New approaches to estimation of white matter connectivity in diffusion tensor MRI: Elliptic PDEs and geodesics in a tensor-warped space. In: MICCAI. (2002) 459-466

5. Jackowski, M., Kao, C., Qiu, M., Constable, R., Staib, L.: White matter tractography by anisotropic wavefront evolution and diffusion tensor imaging. Med Image Anal 9 (2005) $427-440$

6. Fout, N., Huang, J., Ding, Z.: Visualization of neuronal fiber connections from DT-MRI with global optimization. In: ACM Symposium on Applied Computing (SAC). (2005) 1200-1206 
7. Merhof, D., Enders, F., Hastreiter, P., Ganslandt, O., Fahlbusch, R., Nimsky, C., Stamminger, M.: Neuronal fiber connections based on A $^{*}$-pathfinding. In: SPIE Medical Imaging. (2006)

8. Hart, P., Nilsson, N., Raphael, B.: A formal basis for the heuristic determination of minimum cost paths. IEEE Transactions on Systems Science and Cybernetics 4 (1968) 100-107

9. Dechter, R., Pearl, J.: Generalized best-first search strategies and the optimality of A*. Journal of the ACM 32 (1985) 505-536

10. Basser, P., Pierpaoli, C.: Microstructural and physiological features of tissues elucidated by quantitative diffusion tensor MRI. J Magn Reson B 111 (1996) 209-219

11. Essen, D.: A tension-based theory of morphogenesis and compact wiring in the central nervous system. Nature 385 (1997) 313-318 